\title{
Discrete Differential Geometry of Triangles and Escher-Style Trick Art
}

\author{
Naoto Morikawa \\ Genocript, Zama-shi, Kanagawa, Japan \\ Email:nmorika@genocript.com
}

Received 7 February 2016; accepted 2 July 2016; published 5 July 2016

Copyright (C) 2016 by author and Scientific Research Publishing Inc.

This work is licensed under the Creative Commons Attribution International License (CC BY). http://creativecommons.org/licenses/by/4.0/

(c) (i) Open Access

\section{Abstract}

This paper shows the usefulness of discrete differential geometry in global analysis. Using the discrete differential geometry of triangles, we could consider the global structure of closed trajectories (of triangles) on a triangular mesh consisting of congruent isosceles triangles. As an example, we perform global analysis of an Escher-style trick art, i.e., a simpler version of "Ascending and Descending". After defining the local structure on the trick art, we analyze its global structure and attribute its paradox to a singular point (i.e., a singular triangle) at the center. Then, the endless "Penrose stairs" is described as a closed trajectory around the isolated singular point. The approach fits well with graphical projection and gives a simple and intuitive example of the interaction between global and local structures. We could deal with higher dimensional objects as well by considering $n$-simplices $(n>2)$ instead of triangles.

\section{Keywords}

Discrete Differential Geometry, Triangle Mesh, Global Analysis, Singular Point, Penrose Stairs

\section{Introduction}

Discrete differential geometry studies discrete equivalents of the geometric notions and methods of differential geometry, such as notions of curvature and integrability for polyhedral surfaces. Progress in the field is to a large extent stimulated by its relevance for computer graphics and mathematical physics [1]. For example, [2] proposes a set of tools to approximate normal vectors and curvatures on arbitrary triangle meshes which play significant roles in computer graphics [3].

On the other hand, [4] proposes the discrete differential geometry of $n$-simplices and considers flows (of $n$-simplices) on a specific $n$-dimensional mesh. In other words, it considers connection between space-filling $n$-simplices. For $n=3$, we obtain the discrete differential geometry of tetrahedrons which is used for protein 
structure analysis, where protein backbone structures are approximated by a trajectory of tetrahedrons. In this paper, we consider the case of $n=2$. That is, we consider a flow (of triangles) on a triangular mesh consisting of congruent isosceles triangles (Figure 1(a)).

Bringing in a differential structure on the mesh, we could consider the global structure of closed trajectories of triangles. As an example, we consider the paradox of an Escher-style trick art shown in Figure 1(b), a simpler version of "Ascending and Descending". Then, we would associate the paradox with "a trajectory around a singular point".

Ascending and Descending is a lithograph made by dutch artist M. C. Escher, who gets the idea from an article by L. S. Penrose and R. Penrose [5]. In the article they presented an endless staircase called the "Penrose stairs" that continued to ascend and descend, but never got any higher [6]. The Atiyah-Singer index theorem tells us it is an impossible object as indicated on the 2004 Abel Prize announcement [7]. Escher drawings with the structure of the "Penrose stairs" are also mentioned in [8] as the first example of the global/local distinction. However, the Escher drawings are usually described as examples of optical illusion, and detailed mathematical analysis are rarely available. In the following, we give a detailed global analysis of the paradox of the lithograph, using a discrete mathematical approach.

\section{Flow on a Triangular Mesh}

Let's consider a flow of triangles on the mesh shown in Figure 1(a). In our case, a trajectory enters a triangle through a edge and leaves the triangle through another edge. Figure 2 shows an example of step-wise construction of a trajectory on the mesh. Note that there are two choices of moving direction at each triangle: left or right. To indicate the moving direction at each triangle, we use heavy edges as shown in Figure 2(d). That is, heavy edges are the edges of triangles which are not crossed over in the trajectory.

In Figure 2, triangles are assigned only one heavy edge and move smoothly over the trajectory. However triangles might be assigned more than one heavy edges as in the case of the flow shown in Figure 1(b), where the triangle at the center is assigned three heavy edges. See Figure 3 for all the types of triangles which could

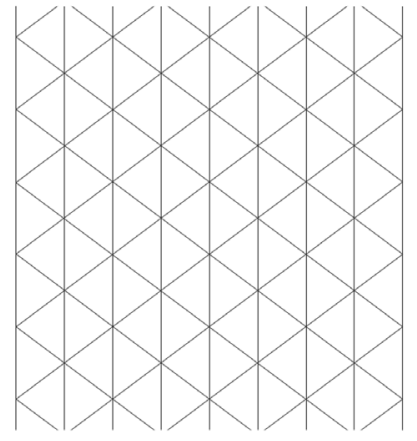

(a)

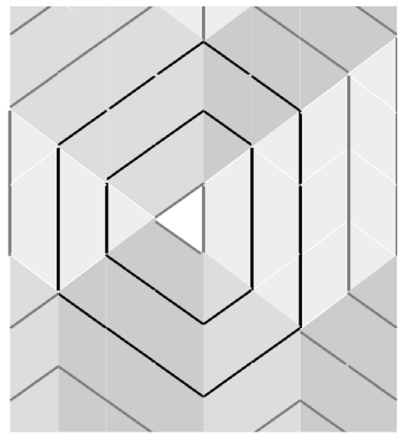

(b)

Figure 1. (a): The triangular mesh, on which a differential structure will be defined. (b): An example of Escher-style trick art (a simpler version of the lithograph Ascending and Descending by M. C. Escher).

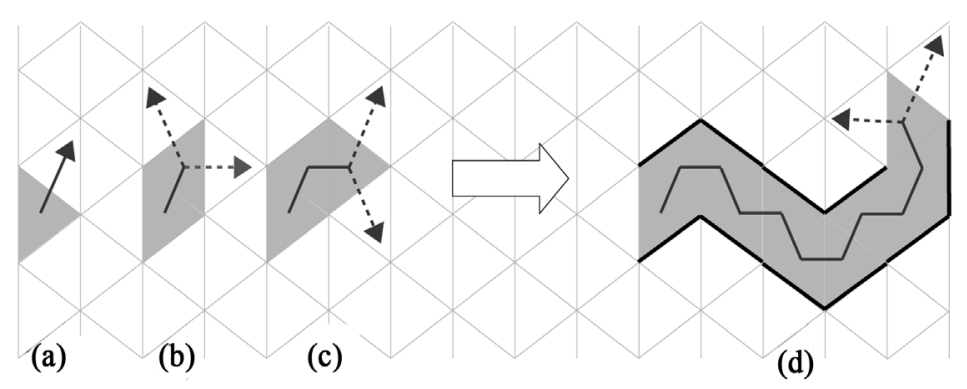

Figure 2. Step-wise construction of a trajectory on the mesh. 
occur.

A regular triangle is a triangle with one heavy edge. A terminal triangle is a triangle with two heavy edges. An isolated triangle is a triangle with three heavy edges. A branch triangle is a triangle with no heavy edge. A singular triangle is a triangle which is not regular.

Assigning heavy edges to all the triangles in the mesh, we obtain a flow of triangles. A flow without singular triangles is called a regular flow.

\section{Differential Structure on the Triangular Mesh}

Discrete differential structure is defined on the triangular mesh to obtain a discrete version of Riemannian manifold as follows. Firstly we consider a three-dimensional cube over the mesh as shown in Figure 4(a). Each of the three upper faces of the cube is divided into two isosceles triangles by a diagonal segment (heavy line). Then, the six triangles on the upper faces are projected onto six triangles in the mesh below. Triangles on the upper faces of a cube is called a slant triangle. Triangles in the mesh are called a flat triangle. See [4] for a detailed description of the tangent bundle-like structure on the mesh.

By piling up these cubes diagonally, we obtain a mountain and valley-like structure, which is called an affine cube covering of the mesh. Regular slant triangles on an affine cube covering are associated with flat triangles in the mesh in such a way that the heavy edges of the former are projected onto the heavy edges of the latter. In particular the gradient of the slant triangle over a flat triangle indicates the moving direction of a trajectory at the flat triangle (note that we could not associate a singular flat triangle with a slant triangle because the latter has only one heavy edge).

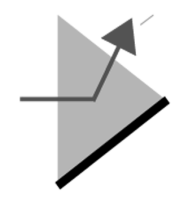

(a)

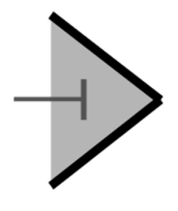

(b)

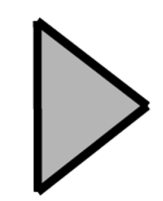

(c)

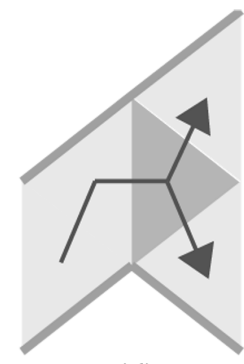

(d)

Figure 3. Regular triangle and three types of singular triangle. (a): Regular triangle. (b): Terminal triangle. (c): Isolated triangle. (d): Branch triangle.

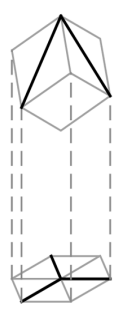

(a)
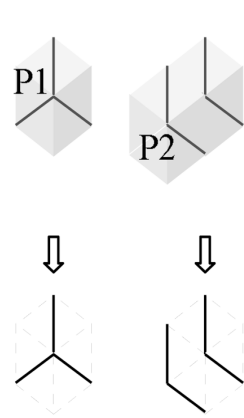
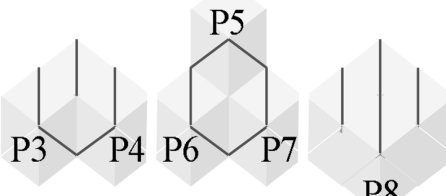

P9 1 P11
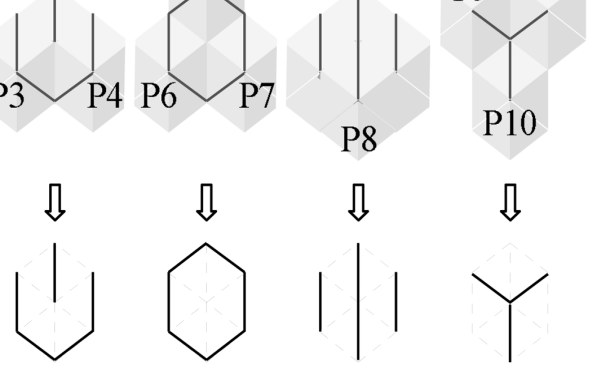<smiles>O=C1CCCCC1</smiles>

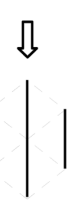

$\sqrt{2}$

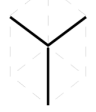

(b)

Figure 4. Local differential structure. (a): Projection of a three-dimensional cube over the mesh. (b): Six regular flat triangles (bottom) and their corresponding slant triangles (top), where faces of three-dimensional cubes (i.e., slant triangles) are colored differently based on their gradient for the 3D shading effect. See [4] for a detailed description of the structure. 
Locally the corresponding slant triangle over a regular flat triangle is obtainable as shown in Figure 4(b). The figure shows all the patterns of the six regular flat triangles in a hexagonal region. The corresponding affine cube coverings are uniquely determined by the collection of their peaks $\{P 1\},\{P 2\},\{P 3, P 4\},\{P 5, P 6, P 7\}$, $\{P 8\}$ and $\{P 9, P 10, P 11\}$ respectively.

As for the problem of extension of a "local" affine cube covering over the whole flow on the mesh, we have the following theorem.

Theorem 1 (Continuation Theorem) An affine cube covering is extendable over a closed trajectory of a regular flow.

See Appendix for the sketch of the proof.

\section{Singular Triangle and Escher-Style Trick Art}

Now let's look at the Escher-style trick art given in Figure 1(b) again. In the language explained above, it is an "impossible affine cube covering" of a flow of triangles which has an isolated triangle at the center. That is, it is the isometric projection of an object (which is obtained by piling up cubes diagonally) onto a plane, where surface color indicates its gradient. Because of the singular triangle, we need more than one cube coverings to cover the closed trajectory surrounded by the black polygonal lines. See Figure 5 for an example of a set of affine cube coverings required to cover the whole trajectory.

\section{Conclusions}

Using the discrete differential geometry of triangles (i.e., 2-simplices) proposed in [4], we have considered the structure of an Escher-style trick art. Our approach gives a discrete version of Riemannian manifold in such a way as to fit well with graphical projection, i.e., the projection of three-dimensional objects onto a planar surface. Moreover, we could deal with higher dimensional discrete objects as well by considering $n$-simplices $(n>2)$. (Recall that researchers in the field of discrete differential geometry mainly study polygonal curves and polyhedral surfaces to establish the smooth theory as limit of the discrete theory)

With this approach at our disposal, the interaction between global and local structures of $n$-dimensional discrete objects $(n>1)$ could be described concisely in an intuitive fashion. As an example, we have shown that

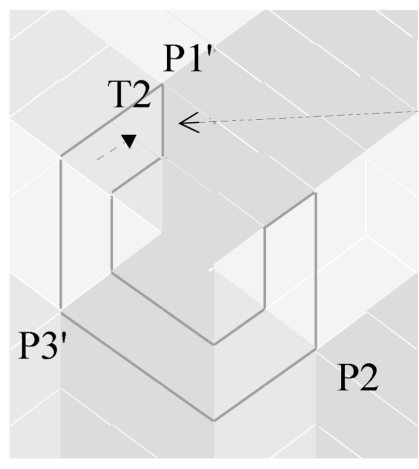

(a)

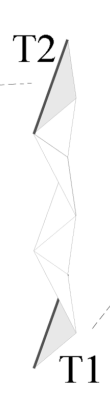

(b)

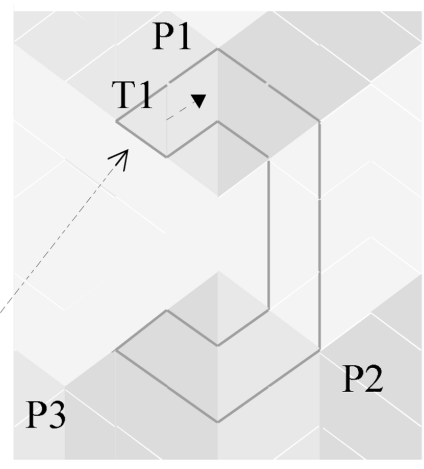

(c)

Figure 5. An example of a set of affine cube coverings over a closed trajectory occurred in Figure 1(b). A local affine cube covering is extended clockwise along the trajectory starting at slant triangle $T 1$ and ending at slant triangle $T 2$. Both $T 1$ and $T 2$ are projected onto the same flat triangle in the mesh. The black polygonal lines indicate the range of the trajectory to be covered. We need more than one affine cube coverings to cover the whole trajectory. (a): Affine cube covering around $T 2$ with peaks $\left\{P 1^{\prime}, P 2, P 3^{\prime}\right\}$. (b): Positional relationship between $T 1$ and $T 2$ in the "tangent space" at the corresponding flat triangle. See [4] for the definition of the tangent space structure. (c): Affine cube covering around $T 1$ with peaks $\{P 1, P 2, P 3\}$. Slant triangles are colored differently based on their gradient for the $3 \mathrm{D}$ shading effect. 
the paradox of the Escher-style trick art is attributable to a singular triangle at the center.

\section{References}

[1] Bobenko, A.I., Schroder, P., Sullivan, J.M. and Ziegler, G.M. (2008) Discrete Differential Geometry. Overwolfach Seminars. Vol. 38, Birkhauser, Basel.

[2] Meyer, M., Desbrun, M., Schroder, P. and Barr, A.H. (2003) Discrete Differential-Geometry Operators for Triangulated 2-Manifolds. In: Hege, H.-C. and Polthier, K., Eds., Visualization and Mathematics III, Springer-Verlag, Berlin, $35-57$.

[3] Desbrun, M., Schroder, P. and Wardetzky, M. (2008) Discrete Differential Geometry: An Applied Introduction. Siggraph Asia 2008 Course Notes, Singapore.

[4] Morikawa, N. (2014) Discrete Differential Geometry of $n$-Simplices and Protein Structure Analysis. Applied Mathematics, 5, 2458-2463. http://dx.doi.org/10.4236/am.2014.516237

[5] Schattschneider, D. (2010) The Mathematical Side of M.C. Escher. Notices of the American Mathematical Society, 57, 706-718.

[6] Penrose, L.S. and Penrose, R. (1958) Impossible Objects: A Special Type of Visual Illusion. British Journal of Psychology, 49, 31-33. http://dx.doi.org/10.1111/j.2044-8295.1958.tb00634.x

[7] Rognes, J. (2004) About the Laureates Work. The Abel Prize Laureate 2004 International Page. http://www.abelprize.no/c53865/binfil/download.php?tid=53804

[8] Franklin, J. (2014) Global and Local. Mathematical Intelligencer, 36, 4-9. http://dx.doi.org/10.1007/s00283-014-9482-0 


\section{Appendix}

\section{Scketch of the Proof of Theorem 1}

Proof. We could construct a cube covering over a closed trajectory in the following four steps.

[Step 1] Choose any triangle $T$ on the closed trajectory and construct a local cube covering $C_{T}$ over the triangle (each triangle in the flow has a local cube covering because the flow is regular).

[Step 2] Extend $C_{T}$ along the closed trajectory to obtain an extension $E_{0}$ over the closed trajectory. Then, we obtain a extension $E_{1}$ over the whole flow by extending the slopes of $E_{0}$ downward (without piling up cubes). The gradient of the slant triangles on $E_{1}$ may not corresponding to the moving direction of the flat triangles in the flow. The flat triangle with contradicting moving direction (w.r.t. $E_{1}$ ) is called contradicting flat triangles below. Let $U$ be the union of the closed trajectory and the area surrounded by the closed trajectory. Let $F_{c o n}\left(E_{1}\right)$ be the collection of the contradicting flat triangles in $U$. See Figure 6 for an example.

[Step 3] Solve local contradictions in $F_{c o n}\left(E_{1}\right)$ by piling up cubes one by one in a bottom-up manner (from the boundary region), and we obtain another extension $E_{2}$ which contains all the "peaks" over $U . F_{c o n}\left(E_{2}\right)$ is defined similarly as $F_{c o n}\left(E_{1}\right)$. (Since the flow is regular, a local covering over a contradicting flat triangle would be obtained by piling up cubes locally. The process will end in finite steps because $F_{c o n}\left(E_{1}\right)$ is finite.)

[Step 4] Solve local contradictions in $F_{c o n}\left(E_{2}\right)$ by piling up cubes one by one in a top-down manner (from the regions around the peaks), and we obtain a cube covering over $U$ (since local modifications of the extension have no influence on the slant triangles above, a local modification would not destroy the modifications done before in the process).

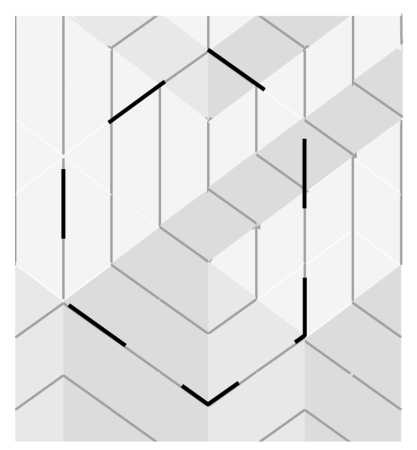

(a)

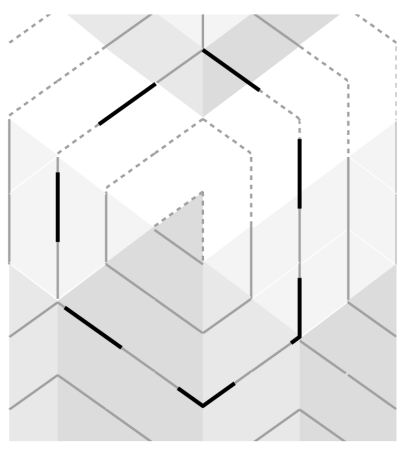

(b)

Figure 6. Extension of a local cube covering along a closed trajectory. (a): An extension of a local cube covering along the closed trajectory surrounded by the black polygonal lines in Figure 1(b). The black broken line indicates the position of the outer boundary of the trajectory. (b): The white triangles show the region where the gradient of the slant triangles on the extension does not correspond to the moving direction of the flat triangles in the flow. The flat triangle at the center has no local cube covering because the flow in not regular. 


\section{Submit or recommend next manuscript to SCIRP and we will provide best service for you:}

Accepting pre-submission inquiries through Email, Facebook, Linkedin, Twitter, etc A wide selection of journals (inclusive of 9 subjects, more than 200 journals)

Providing a 24-hour high-quality service

User-friendly online submission system

Fair and swift peer-review system

Efficient typesetting and proofreading procedure

Display of the result of downloads and visits, as well as the number of cited articles

Maximum dissemination of your research work

Submit your manuscript at: http://papersubmission.scirp.org/ 